\title{
Feature-Based Virtual Engineering
}

\author{
G. BRUNETTI \\ Centro de Excêlencia em Tecnologias Avançadas do Rio Grande do Sul - CETA-RS \\ Av. Assis Brasil 8450, 91140-000 Porto Alegre, RS, Brazil
}

Fraunhofer-Institut für Graphicshe Datenverarbeitung - IGD

Fraunhofer Straße 6, 64283 Darmstadt,Germany

gino.brunetti@igd.fhg.de

http://www.igd.fhg.de/igd-a2/

\begin{abstract}
In this paper the concept of Feature-based Virtual Engineering is presented as an approach to integrated product development. The focus of the paper is on presenting the current state of the development of a feature-based parametric representation for product semantics including a concept for creating and maintaining application specific views on the product information.
\end{abstract}

Key words: Virtual Engineering, Feature-based Product Representation, Integrated Product Development

\section{INTRODUCTION}

Nowadays, the use of virtual reality technology in engineering is mostly restricted to the design review and decision support. Typical VR applications are designed to provide an appealing visualisation of product geometry. Some of these applications support the shared viewing of virtual models in distributed environments. Others allow the animation of dynamic data obtained from, for instance, simulations and kinematics, or they support the simulation of processes like assembling. Emerging technologies like augmented reality for assembly and maintenance are only in a very early experimental phase. What these VR applications have in common is that the interaction with the model very often is limited to navigation and the release of predefined events. They usually do not allow the generation or modification of product information. 
In order to achieve a further-reaching integration of VR in the product development process it is necessary to develop today's VR environments that are engineering data dead-ends into human centred interfaces of real Virtual Engineering (VE) environments. Such VE environments have to provide access to all available product information at the time required. The presentation of product information has to be configurable depending on the task that has to be performed. In addition to the traditional presentation of digital products VE environments have to allow to efficiently enter and modify product information. It must be possible to examine not only the physical aspects of products, but also to experience its function by transforming functional product information into corresponding interactive behaviour. Intelligent interaction methods have to be flexibly configurable depending on the user characteristics, the current task, the relevant information, and the I/O-devices available. Furthermore, VE systems have to provide intelligent feedback and guidance in order to support users as far as possible in achieving "correct" results. Finally, both the interaction control as well as the presentation of the product information must be automatically configured based on an interpretation of the product semantics instead of the manual scene configuration as it is usually done today. In this way, VE increases the intuitiveness of interaction with the digital product and raises the acceptance of the use of VR technology in engineering.

Feature-based VE environments as introduced in this paper are intended to overcome the disadvantages of today's approaches to an integrated product development that are based on data driven methodologies: the use of direct converters to exchange product data between highly specialised legacy systems; information lost or faulty information because interpretation of data is not stored within the product data structure, but interpreted by the various systems differently; insufficient support of modification back-propagation; and, integration only on file level. The introduced approach to VE in turn is based on an integrated product data structure that is feature-based and potentially able to store all relevant product information as well as to capture a high level of engineering semantics. In this approach product information is structured vertically according to different levels of semantic abstractions and horizontally according to the processes of the product development they belong to. Relationships between information units describing the different types of geometric as well as non-geometric properties of a product are managed via constraints and rules defining the knowledge-base about a product and all related processes.

For users the concept to access and manage product information is based on dynamically configurable views. Such views are defined by system configurations, by process information specifying the relevant product information, and by the filters and converters interfacing a VE environment 
with other systems producing and consuming the different types of product information. Furthermore, views are a means of abstraction allowing users to easily map their engineering know-how into a corresponding representation of product semantics in terms of meaningful features. Finally, it is the author's belief that feature-based virtual engineering as described above significantly improves the man machine interaction by providing highly sophisticated means of interaction with a digital product and all relevant meta

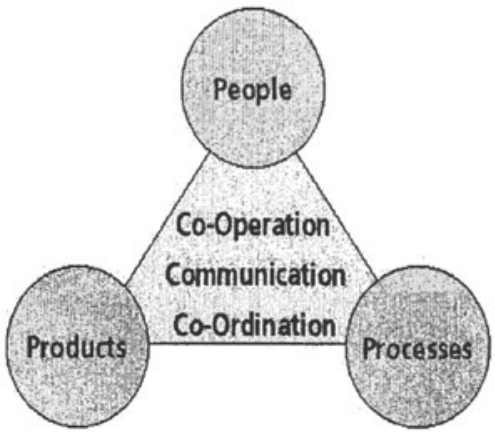

Figure 1. Elements of Virtual Engineering data.

The paper is organised as follows. First the basic idea of virtual engineering is described. Afterwards, the requirements on a feature-based architecture for a Virtual Engineering environment supporting different views on a product is presented. This follows an introduction into the used feature definition and a description of the feature-based product data model including views, which is also the basic content of this paper. Guided by the systematic approach to engineering design illustrated in Figure 7 the discussion starts with the detail design phase followed by a bottom down approach from conceptual design to embodiment and assembly design.

\section{VIRTUAL ENGINEERING}

The implementation of the Virtual Engineering (VE) concept needs the integration of methods and tools, which are able of working in heterogeneous distributed system environments (de Amicis et al. 1999). In addition different visualisation techniques are used to support the different phases of the product development process in a goal-oriented way. The possible visualisation hardware spans completely different equipment such as the monitor in the office, a virtual table for working and management meetings, a large screen projection for the presentation of models and products in real-scale, or even a five sided CAVE.

The actual research activities in the field of information and communication technology lead to short intervals of technological innovations. A special interest can be found in the evolution of applications supporting simulation and visualisation of complex interactions in early 
states of the product design process so that the various aspects of a product can be analysed and optimised. In this context VE, for instance, allows the design review of products on basis of CAD data. Furthermore, process planning, production system planning and complex production facilities can be simulated, visualised and evaluated by applying VE technologies.

Besides these technology aspects VE concentrates on the relations between information technology and working environments. Therefore, a special focus is on the harmonisation of the technologies with the corresponding 3Ps: People, Processes, and Products as shown in Figure 1.

Nowadays, the product development is divided into several tasks related to the development competence of each partner. Process chains become more and more integrated and interdisciplinary. Therefore, VE applications must address the need of dealing with engineering teams where the members belong to different organisational units at distributed locations, where integrative processes supporting concurrent engineering even for interdisciplinary tasks are performed, and where various cultures regarding language, mentality, and working approach can be found.

The focus of this paper is the representation of product semantics that supports VE applications allowing users to interact and to experience the digital product according to the product information available along the product life-cycle. As outlined in the following sections the presented approach manages such product semantics within a parametric feature model.

\section{FEATURE-BASED VIRTUAL ENGINEERING}

It is generally agreed that computer supported engineering environments have to manage a much broader range of information than geometry. Besides other aspects it becomes more and more crucial to capture, represent and process the function and the functional behaviour of a product. This should already be done in the early phases of the product development process. Furthermore, designers should be assisted in developing syntactical correct and semantically consistent product models by providing intelligent and intuitive computer support that minimises the possible sources of engineering errors and maximises the capability to map designers' creativity into corresponding mathematical models of the product. The presented approach to these objectives is a feature-based representation scheme for product semantics including product function. Features are then the information carriers that allow modelling the relationships between requirements, functional descriptions and physical solutions of a product. They become the vehicle to bring this information to the downstream 
applications for embodiment, analysis, detail part design, and assembly such that it is possible, for instance, to keep track of the consistency between the concept, the design, and the manufacturing of a product. Approaches existing towards a feature-based integration of different phases of the product development to support concurrent engineering, which include previous work of the author, are based on extracting and mapping different views of a parametric representation of a product that are expressed in terms of geometric form features where products semantics are expressed in terms of explicit geometric or functional constraints (de Martino et al. 1998, Brunetti et al. 1996, de Kraker et al. 1995). Product semantics as it is handled within the conceptual design is not supported. Therefore, it is the aim of this paper to come up with a concept to extend these feature-based integration approaches in a way that it is possible to represent the conceptual design information, to support the evolution of product semantics along the product development process, and finally to improve concurrent engineering and top-down design. This is done by supporting an early feature-based prototyping of the different views to the overall product model making use of captured feature information to the largest extend possible. Major prerequisite of such an approach and therefore focus of this paper is a suitable representation scheme to store, manage, and retrieve product semantics and views on them.

\subsection{Feature definition}

The feature definition adopted in the presented work is that of the FEMEX (Feature Modelling Experts) working group established in 1996 by a number of international researchers (including the author), CAD system developers and larger CAD user companies (see, for instance, Vajna and Podehl 1998). According to the results of FEMEX a feature is defined as follows:

A feature is an information unit (element) representing a region of interest within a product. It is described by an aggregation of properties of a product. The description contains the relevant properties including their values and their relations (structure and constraints). Furthermore, it is defined in the scope of a specific view onto the product description with respect to the classes of properties and to the phases of the product lifecycle. Finally, a feature is described by properties out of several different classes of properties, thus relating these (classes of) properties to one another. 
There are five major aspects in the definition above:

1. It is necessary to find a structure of properties suitable to express relevant product information in the different phases of the product life-cycle in terms of such product properties.

2. A feature is not limited to physical elements and exists only in the world of information models.

3. "Properties" are fundamental to the definition and at the same time the basic implementation mechanism.

4. The concept of views is inherent to the definition.

5. "Classes of properties" and "product life-cycle phases" are distinguished in the definition above. Some properties are meaningful in more than one phase, for instance, geometry - in different phenotypes - is considered in nearly every phase of the product life-cycle. The product information expressed in terms of properties aggregated by different features in different application contexts (views) is therefore the key mechanism towards an integrated product development.

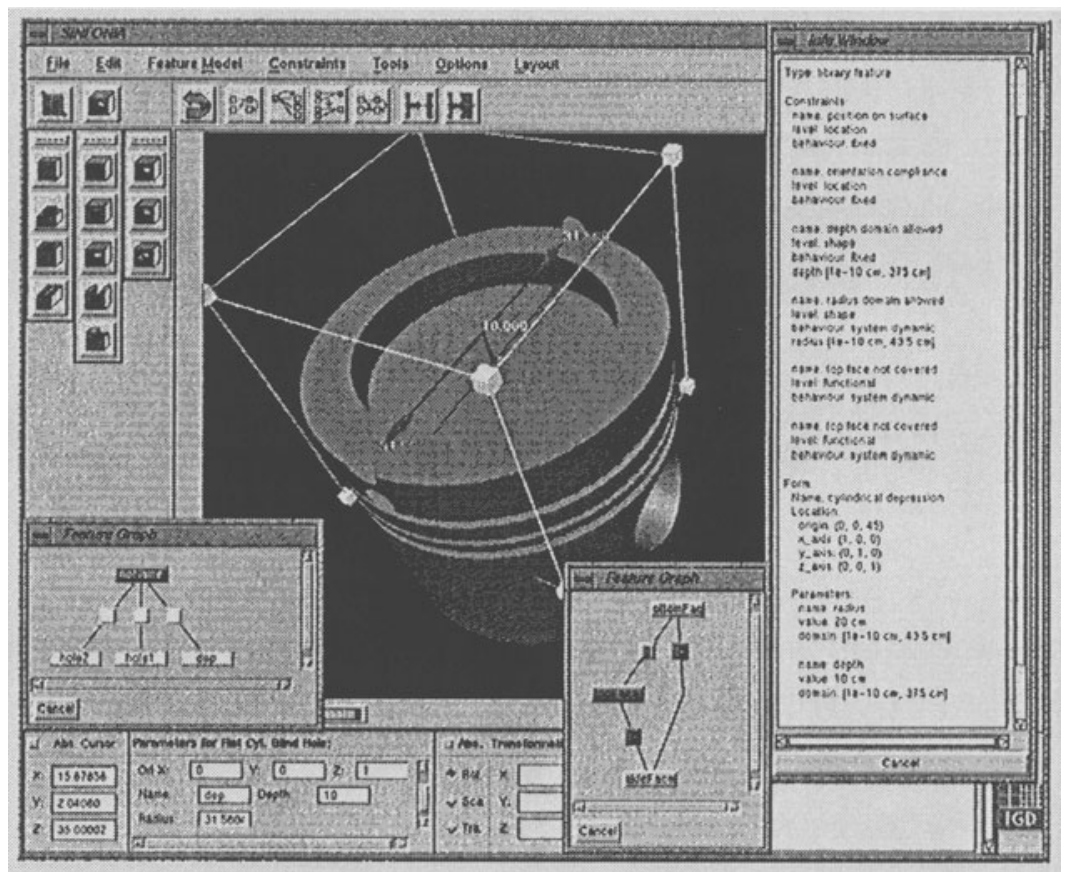

Figure 2. The feature-based parametric modelling system SINFONIA. 


\subsection{Feature-based parametric product Representation}

The conceptual starting point and basis for the implementation of the presented approach towards an feature-based virtual engineering environment is the prototype system SINFONIA (see Figure 2). The latest results obtained with this system in the area of feature-based part and assembly modelling have been published in (Brunetti and Stork 1998). SINFONIA's underlying approach combines feature-based and parametric modelling methodologies and, following the FEMEX feature definition, structures feature data into four levels: an assembly model, a part model, a feature model, and a generic model. Starting with the assemblies every representation level is mapped on a model of the subsequent level, i.e. elements of a higher level are modelled by elements of the lower levels.

Application specific data describing a product assembly and its parts are specified in the first three models of the representation that consists of an assembly model, a part model, and a feature model. A feature model is built on top of the generic representation, which stores application independent product properties within a generic model. The data maintained in this generic model so far specifies the form and parametrics of the parts as well as the geometric and topological constraints defined on them. The final shape of the parts and their relative position within the assembly is derived from this generic model and mapped on a solid modeller.

\subsubsection{Parametrics of the generic model}

The generic model is an extended semantic net. Its nodes are topological form feature entities, geometric feature entities, or parameters. Arcs are constraints. It is a parametric model extending the declarative approach to feature-based modelling. Each form entity is always associated to its correspondent geometric description. Geometric entities are linked to each other by several types of spatial relations expressed as geometric constraints. The third type of entities are the geometric and functional parameters that can be constrained.

The spatial dependencies between features and their geometric entities are described by geometric constraints. Geometric constraints are, for instance, coincidence, complementarity, parallelism, intersection, perpendicularity, concentricity, and coaxiality. Parameters are controlled by domain and value constraints. Variations can be performed linearly, in equidistant steps, or by selecting values out of a concrete set. Interaction constraints specify the way form entities of features behave in relation to their intersection, attachment, and covering. Engineering constraints control 
the value and behaviour of parameters. They are expressed by analytic equations and build a system of non-linear equations. Using such constraints, parameter values are propagated, which implies the use of suitable solving techniques. Other engineering constraints are Boolean expressions which are used for checking logical conditions between parameter values.

\subsubsection{Feature model}

Application specific features and feature relationships build the feature model. Features contain their geometric, parametric, and functional description. They are related to generic objects representing the geometric and non-geometric properties of the feature. Feature parameters result from relationships established between different features of the model. Feature relations specify relative positions and parametric dependencies between features. They can be any non-empty aggregate of the supported constraints (see Figure 3 and Figure 4).

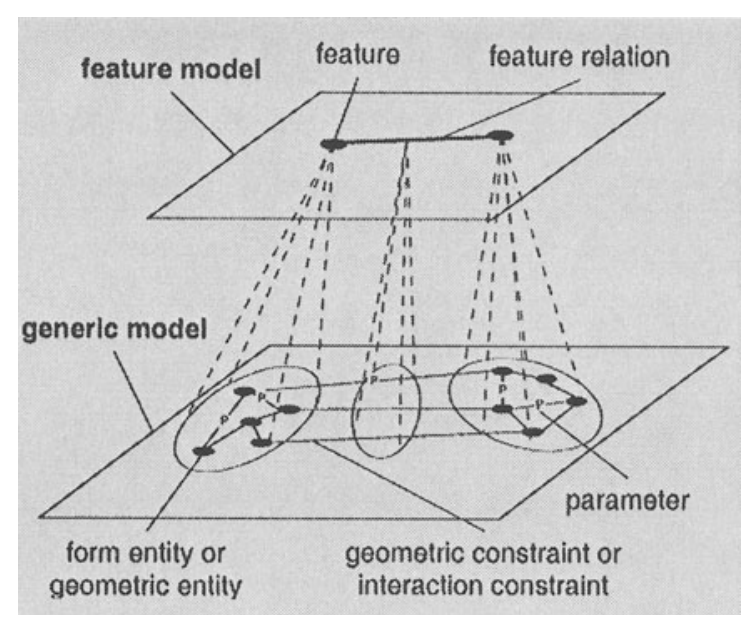

Figure 3. Mapping between a feature and its generic model. 


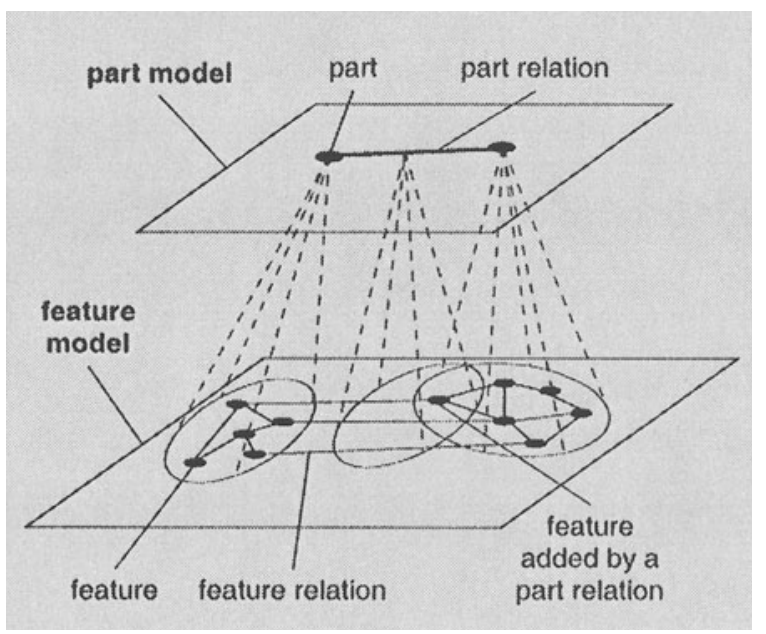

Figure 4. Mapping between a part and its feature model.

\subsubsection{Part model}

Parts, part parameters, and their relation-ships are kept in the part model that also builds a graph structure. A part node contains the functional description of a part from which the feature-based representation is derived. Part parameters are the parameters with a direct link to a corresponding feature parameter, but also part specific parameters as the material or the designer of a part. A part relation allows to specify a geometric or nongeometric detail of the relationship between two parts in an assembly. A geometric relationship between two parts is defined by instantiating a set of suitable feature relations between features of the parts (see Figure 4). If necessary, the features needed to establish the part relation are inserted in the feature model of the parts. Part relations then control the relative position of the parts and the dimensioning of the features involved in the part relations. Non-geometric part relations include, for instance, information about material compatibility's, needed tools, and installation space. 


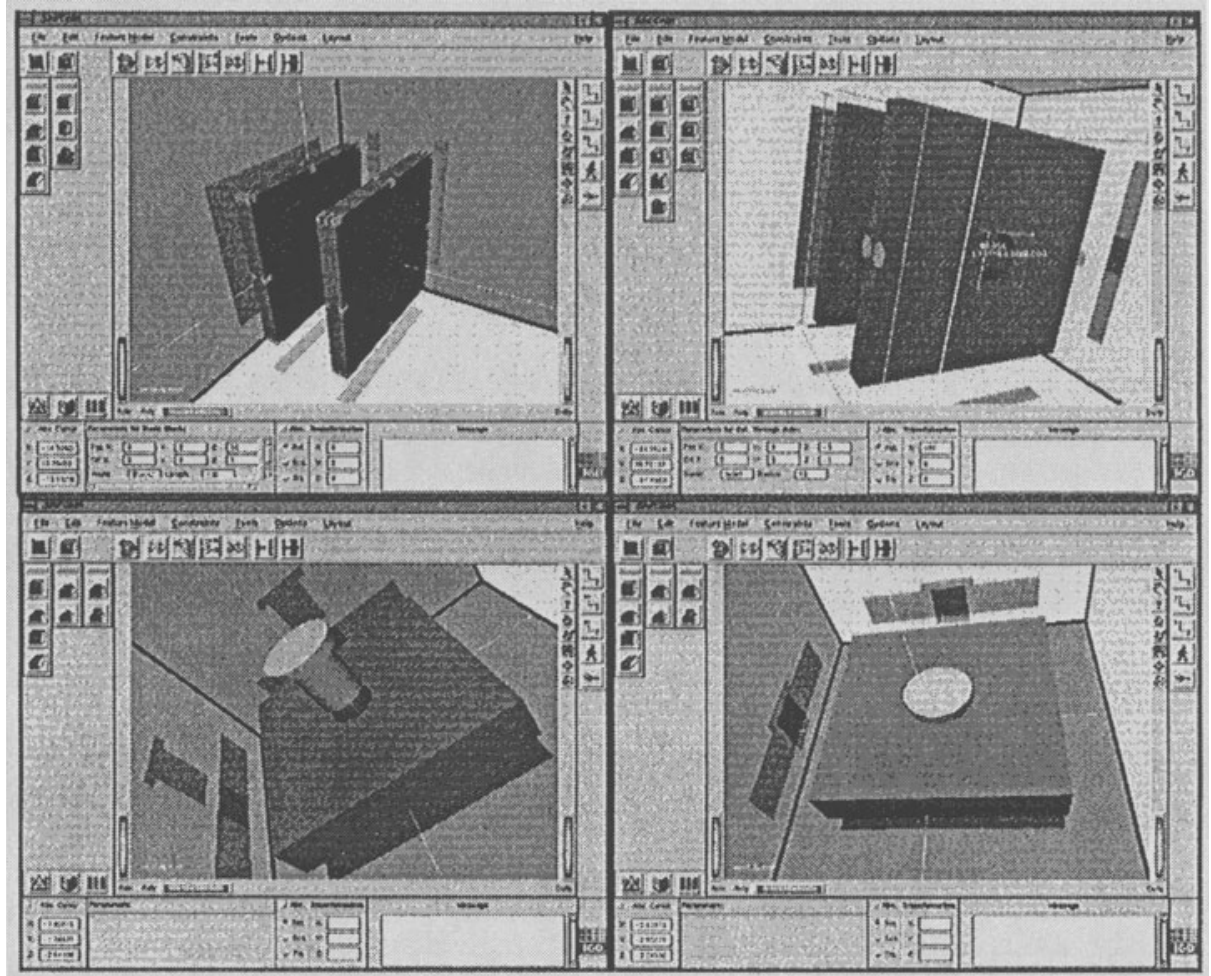

Figure 5. Attaching two plates by a bolt assembly within the SINFONIA system.

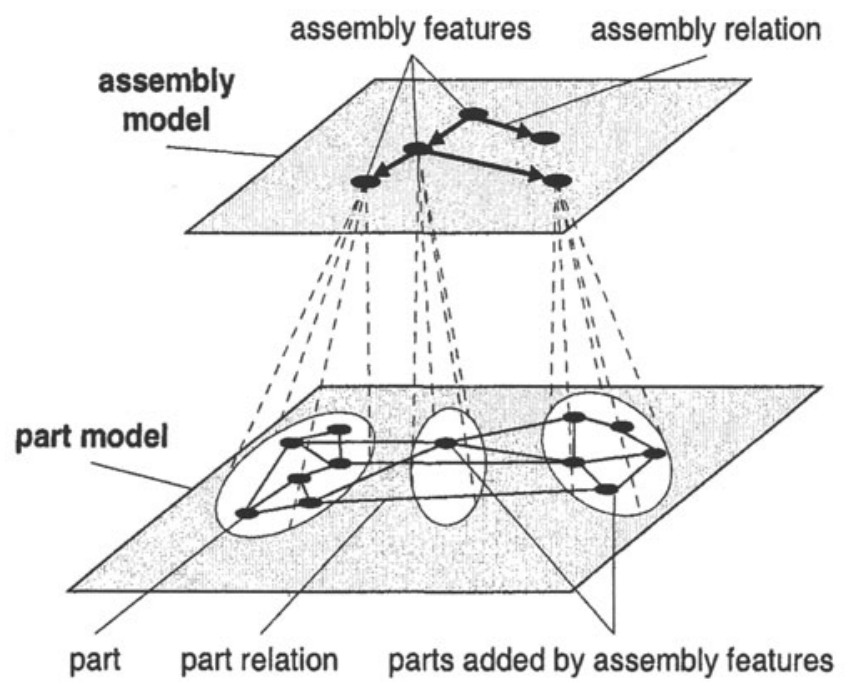

Figure 6. Mapping between an assembly and a part model. 


\subsubsection{Assembly model}

The assembly model is organised as a tree with nodes representing assembly features and edges representing the assembly order. In addition to traditional assembly representations, assembly features contain not just the assembled parts or sub-assemblies of a product, but also the semantic of the relation between the parts. This semantic is part of the assembly feature description and includes the requirements on the parts to be assembled as well as all structuring information of the final assembly. Assembly features add part objects to the corresponding part model. These objects can be parts as, for instance, the screw of a screwing assembly, but are mainly a set of part relations. The relation between an assembly model and the subsequent part model is illustrated in Figure 6. See also Figure 5, which shows the current functionality of our prototype system SINFONIA. In the example two mechanical parts - plates - are connected by a mating part relation and by a bolt assembly feature that inserts two through holes to the model and creates a bolt to assemble the parts.

\subsubsection{Feature-based conceptual design}

Recently, the above described representation scheme of SINFONIA has been extended to the requirements of a more complete product data scheme extending the approach to the early phases of the design process, i.e. conceptual and embodiment design (Brunetti and Borut 2000). Following, the basic information units managed are shortly described and it is outlined how they have been inserted into the over all feature representation scheme.

All design starts with a requirements list that results from the very first task clarification phase (see Figure 7). It describes the general constraints a product has to or should fulfil. An important prerequisite to enable automatic validation of proposed solutions against given requirements is a structured and formalised description of these requirements that has to handle product function, effects, principal solutions, working principles, and finally also embodiments.

\subsubsection{Product function}

Technical systems perform the conversion of energy, material and/or signals. This conversion can be described as flow through the system, where one is the main flow and others, if any, are supporting flows. For the design of a technical system, a clear attitude between the input and output must be defined in form of the function of the system. 


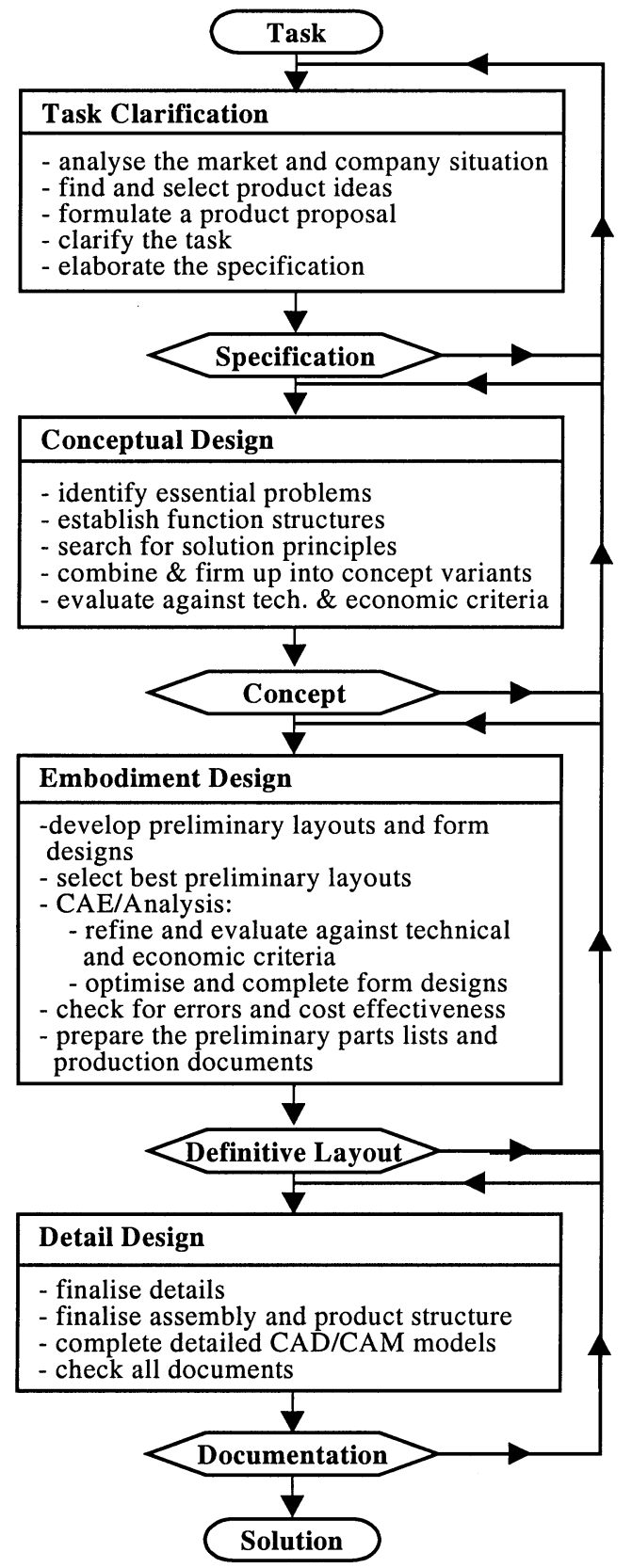

Figure 7. The design process according to the systematic approach to engineering design by Pahl and Beitz 1996.

This function is an abstract formulation of the task. The overall function can often be divided into sub-functions, and function structures can be 
established. With further sub-division of the sub-functions, basic functions also called generally valid - can then be recognised. These functions, according to the definition by Rodenacker and Krumhauer (see, for instance, Pahl and Beitz 1996) are change, vary, connect, channel and store. They represent a conversion of type, magnitude, number, place and time, respectively. Functions are usually fulfilled by physical, chemical or biological processes, whereas mechanical engineering solutions are based mainly on physical processes. Selected physical effects and the determined material and geometric characteristics result in a working principle that fulfils each function. If a function cannot be fulfilled with a simple effect, a structure of effects has to be used instead. In the following phase of the design process - embodiment - qualitative and quantitative parameters such as surfaces, dimensions and material properties are specified according to the physical laws defining the effects.

\subsubsection{Working principles}

Figure 8 shows the mapping of a function structure into a working principle. To fulfil the main function of a product, an appropriate principle solution has to be selected. As described later, a principle solution is defined by an effect, an effect carrier, properties and physical laws. To perform the main function, auxiliary functions may be needed to supply supporting flows, to eliminate side effects or to meet given requirements (for example, in order to convert water into steam, an additional flow of energy is necessary). Auxiliary functions could be solved by the same or by additional principle solutions. Therefore, the functional structure has to relate subfunctions with principle solutions.

Physical effects are realised by the working geometry and by working motions. Working geometry is defined by arrangements of working surfaces. These can be varied with respect to and determined by type, shape, position, size and number. Type, nature, direction, magnitude and number determine working motions. Finally, basic material properties are defined by their state (solid, liquid, gaseous, space), their behaviour (rigid, elastic, plastic, viscous), and their form (solid body, grains, powder, dust). 


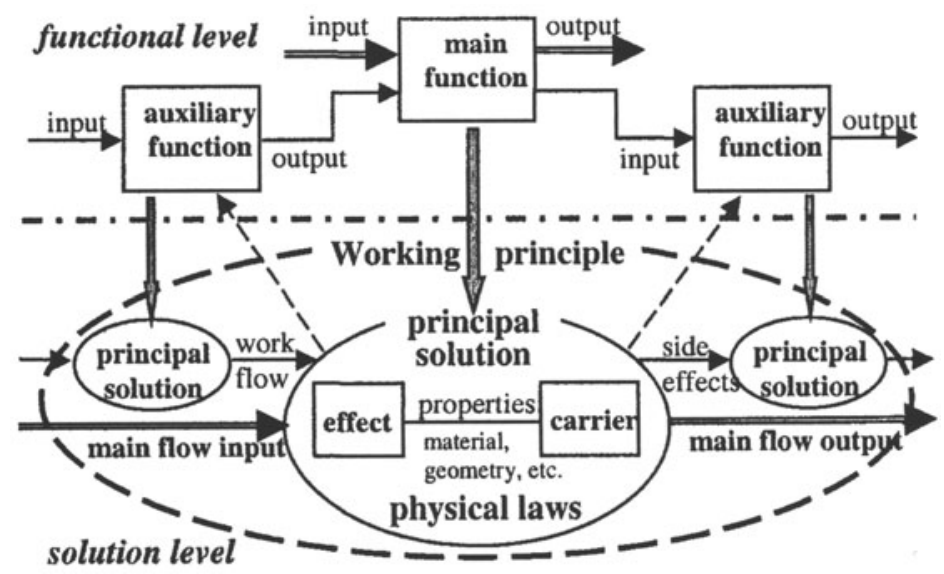

Figure 8. Function fulfilled by a working principle.

\subsubsection{Function model}

A feature-based representation scheme for capturing the product function must provide a means of explicitly modelling the sub-function structure as illustrated in Figure 9. Within such a function model, functions are represented by function features, which not only represent the static function information mentioned above, but also carry the knowledge about its intent and its concretisation in terms of principle solutions.

A principle solution consists of a chosen effect and the appropriate effect carrier. Usually, the same effect can generate several principle solutions, depending on material and geometrical properties; for example, the thermal dilatation effect can be combined with a solid body or a fluid as the carrier. The relation between function and principle solution is normally not one to one. Sometimes, one principle solution can solve several functions at once, however, a lot of functions cannot be solved by only one principle solution. In this case, a principle solutions structure is needed, which is the working principle. 


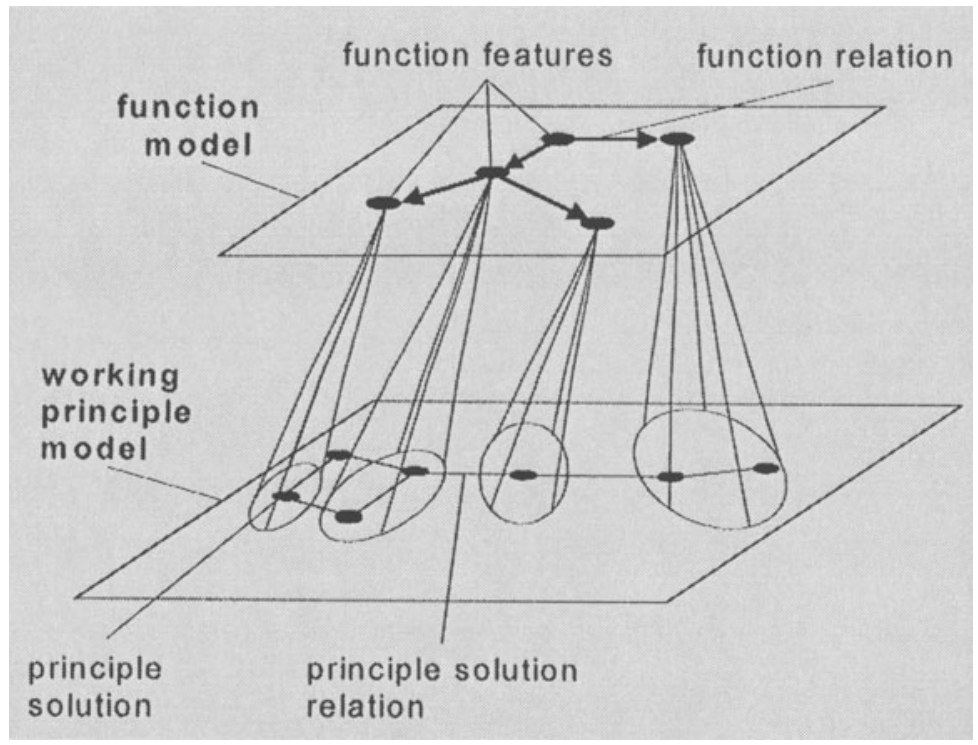

Figure 9. Relation between a function model and a working principle model.

\subsubsection{Working principle model}

As the number of known physical effects is deterministic, some authors have collected related principal solutions for use in the design process (Koller and Kastrup 1988). These catalogues of principle solutions are structured appropriately for a computer implementation (see, for instance, RPK 1999).

An appropriate structure of principle solutions, which fulfils the main function of a product, is a working principle. Together with given requirements, a working principle determines the embodiment of a product. Due to links/connections to auxiliary functions and a hierarchical structure of data, a working principle describes the principle solutions structure and implicitly also the function structure.

In the introduced representation scheme the principle solutions and their inter-relationships in terms of input/output parameters and their properties that make up a working principle are kept within a working principle model, where each principle solution is connected to the one ore more functions it realises. In this way it is possible to establish a parametric relationship between this working principle model and the function model as illustrated in Figure 9. 


\subsection{Feature-based embodiment design}

Finally, the embodiment represents the materialisation of a concept where the overall layout of a product is determined. Embodiment is defined by geometrical properties - shapes, and properties of material from which part or parts are built. Both are influenced by a working principle and requirements. Shapes consist of surfaces - working surfaces, required by working principle and free surfaces. General dimensions are defined either by physical laws governing various effects used in working principles, or from requirements, or by material properties according to a strength and stress analysis, for instance. Principle solutions, as mentioned above, are defined by effects and effect carriers, where each effect carrier has to be considered as a starting point for detailing the layout of an assembly that, once the design is finished, has to have a representation in the assembly model of a product. Therefore, to capture the assembly information obtained during the embodiment design phase, the previously introduced assembly model is extended and related to the working principle solutions model as illustrated in Figure 10. The extensions are a means of representing the product structure and critical relations between assemblies instantiating the parametric relations between principle solutions, which are either basic functions or physical laws manipulating and controlling the input/output parameters of the functions and their principle solutions, respectively.

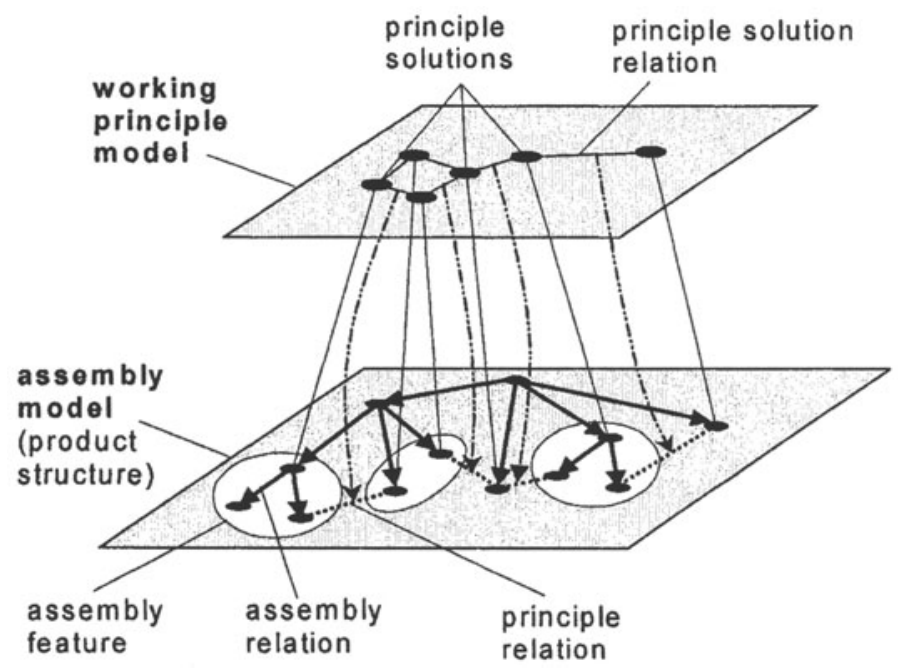

Figure 10. Mapping between a working principle model and an assembly model. 


\subsection{Views}

From a certain perspective the different models defined in the previous section already define particular views onto product data. Nevertheless, views should be understood as a more general concept where any application used within the product development process defines its particular view to the product data. The goal is to come up with a mechanism that allows such applications to extract the required product information, to modify or create new product information, and to merge such information back to the all over product data in a consistent way.

An important prerequisite in order to enable the extraction of different application views to the product information is that this information is stored in small pieces, which are called atoms within this sub-section. Such atoms may be points and lines, but can be also free-form surfaces, a feature, a part, or a function. These atoms are related to one another forming a graph representing the basic knowledge about a product. Such a representation is called an associative or semantic net. The atoms are therefore nodes of a graph. A semantic net is a rather general means of representation. It is limited by the fact that only binary relations are allowed. E.g. the fact that five curves belong to a face of a solid cannot be directly expressed. This restriction is easily removed if general relations are allowed to be represented as atoms. Doing this systematically leads to the concept of conceptual graphs, which are semantic nets with two kinds of nodes. One kind represents the atoms, the other kind relations between atoms, such that the relations of the conceptual graph do not bear additional information. The current implementation of SINFONIA is based on the conceptual graph approach, which is in particular suitable for a complete object-oriented way of implementing both nodes and relations as classes.

However, a user normally does not enter atoms or relations between atoms directly, but she/he will use modelling operations at a higher level, which will generate many atoms and relations during one single operation (see, for instance, the description regarding the part relations). Even a simple modelling operation like „drilling a hole“ generates geometric atoms, topological atoms, functional atoms and possibly administrative atoms, which help to structure the information. Therefore, it is important that a user can look at and even modify that model in different views. A view is a way of structuring and displaying the information stored in the semantic net.

Now, by collapsing sub-graphs according to certain rules (graphmorphisms) different views on the product are provided. The resulting graph structures need not be explicitly stored in the system. E.g. it is not necessary to store an object representing a part with all its relations explicitly; the relations of a part should be consequences of the relations between the atoms 
that constitute the part. Thus the relations could at least in principle be calculated on the fly. It is left to the implementation, whether redundant information is stored or a calculation is performed. Features are identified with the morphisms mapping the atoms in the graphs, which describe the views.

Furthermore, views on the product data must be unique and have to be related to a specific phase (scope within the product life-cycle), version (modifications along the time) and configuration (alternative solutions at a given time) of the product data. This adds a requirement to the VE environment. This requirement is to support product data management functionality for all types and levels of product information. This finally also includes the views themselves.

More formally a view is a function mapping a subset of the product information (a specific version and configuration of atoms and atom relations within a specific scope defined by a phase) into a concrete product data representation that is itself part of the product data model.

The tools/algorithms to generate these views, i.e. map between concrete views, have to fulfil some requirements:

a) Mappings between views can be bijective, as in the case of mappings between different CAD systems, but they have to be at least surjective.

b) Conversions between views like $\mathrm{CAD} / \mathrm{CAE}$ or also CAD/VR are partially injective, i.e. not all information is used for the conversion.

c) Filters are partially surjective, as they filter subsets of the product data model.

d) It should be supported to combine (cascade) mapping functions (A to B to $\mathrm{C}$, if $\mathrm{A}$ to $\mathrm{C}$ is not defined or available).

Associativity between a concrete representation and the product data model is given, if in the case of partially injective mappings modifications on the data must be propagated back to the product data model and all other related views.

It follows that views should be managed within a model of views which itself is part of the product data model. Three major operations should be defined on this model (see Figure 11):

1. The generation of a concrete view out of the product data model, i.e. to generate a derived data model from the original data model.

2. The migration between concrete views, i.e. to map between derived data models (if necessary, via the original data model, i.e. the product data model).

3. The propagation of modifications between views, i.e. the update of the original product data model as well as the active derived (concrete) data models. 


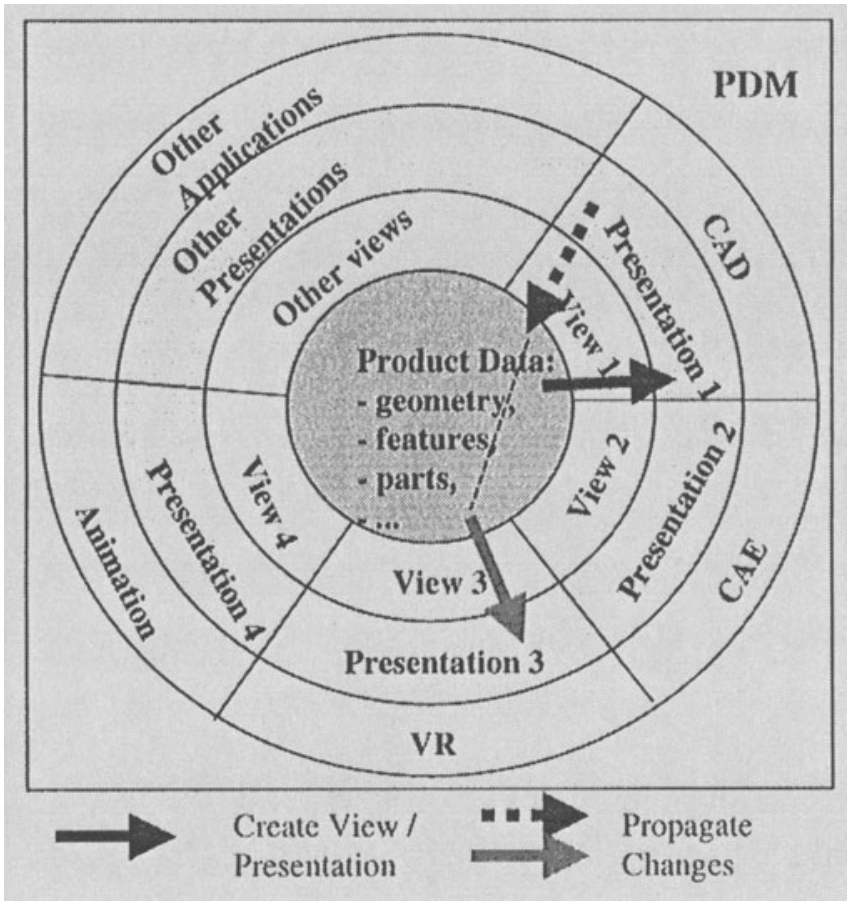

Figure 11. The concept of views.

The advantages of such an approach to keep a central product data model are that all applications of the VE environment work on the same database and that modifications performed by one application are visible to the other applications. Furthermore, by providing a mechanism to specify new mappings for views the system is open and extendable. Finally it takes into account the time dimension in case of product data revisions. The approach could be implemented, for instance, within a product data management system that provides functionality to manage versions and configurations, but on any atom level as presented above rather than on the CAD model level, as it is usually done today.

\section{CONCLUSIONS AND FUTURE WORK}

The work presented summarises the current results of the author's ongoing work towards feature-based Virtual Engineering. The current state of development regarding feature-based assembly modelling with SINFONIA has been presented. Furthermore, extensions of the product data model based on features to capture the relevant product semantics of the early design phases as well as a concept of views has been introduced. 
Guiding principles of the work was the systematic approach to engineering design processes as proposed by Pahl and Beitz, which is a widely applied methodology within the European design schools. Another guideline of the work is the FEMEX feature definition as it is general enough to be applied for the scope of the presented topic.

The major concern for the time being is to efficiently represent the complex inter-relationships of products information in order to describe product semantics and views on them. Currently the possibility to model the presented parametric feature representation in terms of chain models on cellular complexes as introduced in (Palmer and Shapiro 1993) is being analysed, which could provide both an attractive formalisation and a mathematical sound computational model. Furthermore, this would allow to include dynamic physical behaviour into the representation and evaluation of product semantics. Nevertheless, it is the final goal to realise a humanoriented system that aids the creativity of designers in VE environments. Current development activities are in the area of feature-based styling of free form surfaces in VR, which has been motivated by the work presented in (Fontana et al. 1999). Finally, the view concept as presented in this paper will be further elaborated.

\section{REFERENCES}

de Amicis, R. Brunetti, G., Rix, J. (1999). Virtual Engineering and Using Virtual Reality for Engineering, Proceedings of the ADM'99, Palermo, Italy

Brunetti, G., Borut, G. (2000), A Feature-based Approach towards an Integrated Product Model including Conceptual Design Information, Computer-aided Design, Vol. 32 (14) pp. 877-887

Brunetti, G., De Martino, T., Elter, H., Falcidieno, B. (1996), Modelling Shape and Semantics through an Intermediate Model, Proceedings of the 29th Symposium on Automotive Technology \& Automation (ISATA '96), Florence, Italy, pp. 71-81

Brunetti, G., Stork, A. (1998). Product-centered Intituitive 3D Interaction for Feature-based Parametric Assembly Modelling, Proceedings of the ProSTEP Science Days '98, Product Data Technology Facing the Future

Fontana, A., Giannini, F., Meirana, M. (1999). A Free Form Feature Taxonomy, In Brunet, P, Scopigno, R.(eds.): Proceedings of the EUROGRAPHICS'99, Volume 18, Number 3

Koller, R. and Kastrup, N. (1998). Prinziplösungen zur Konstruktion technischer Produkte, 2. Auflage, Springer

de Kraker, K. J., Dohmen, M., and Bronswoort, W. Y. (1995). Multiple way feature conversion to support concurrent engineering, Proceedings of ACM Solid Modelling '95, Salt Lake City, Utah, pp. 105-114

de Martino, T., Falcidieno, B., Haßinger, S. (1998). Design and engineering process integration through a multiple view intermediate modeller in a distributed objectoriented system environment, Computer-Aided Design, Vol. 30, No. 6, pp. 437-452 
Pahl, G. and Beitz, W. (1996). Engineering Design, A Systematic Approach, 2nd edition. London: Springer

Palmer, R. S., Shapiro, V. (1993). Chain Models of Physical Behavior for Engineering Analysis and Design, Research in Engineering Design 5, pp. 161-184

RPK [Institut für Rechneranwendung in Planung und Konstruktion, Karlsruhe(TH)]. Effektdatenbank [online]. (1999), http://rpkalf4.mach.unikarlsruhe.de/ michelis/effects/

Vajna, S. and Podehl, G.(1998). Durchgängige Produktmodellierung mit Features, CADCAM Report Nr. 3, pp. 1-8. 